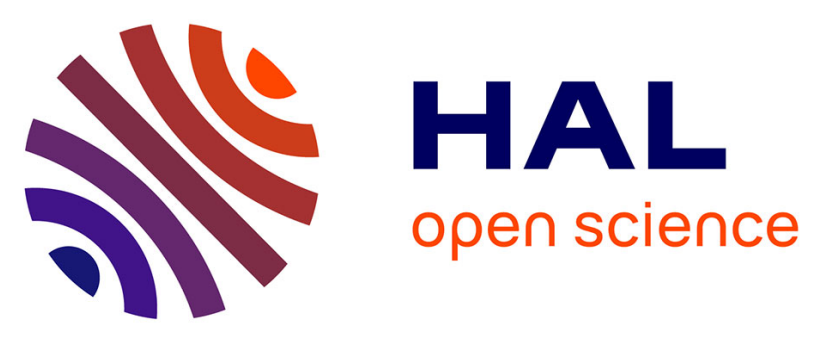

\title{
Transcriptional profiling of immune and inflammatory responses in the context of SARS-CoV-2 fungal superinfection in a human airway epithelial model
}

Claire Nicolas De Lamballerie, Andrés Pizzorno, Julien Fouret, Lea Szpiro, Blandine Padey, Julia Dubois, Thomas Julien, Aurélien Traversier, Victoria Dulière, Pauline Brun, et al.

\section{To cite this version:}

Claire Nicolas De Lamballerie, Andrés Pizzorno, Julien Fouret, Lea Szpiro, Blandine Padey, et al.. Transcriptional profiling of immune and inflammatory responses in the context of SARS-CoV-2 fungal superinfection in a human airway epithelial model. Microorganisms, 2020, 10.1101/2020.05.19.103630 . hal-02988343

\section{HAL Id: hal-02988343 https://hal.science/hal-02988343}

Submitted on 4 Nov 2020

HAL is a multi-disciplinary open access archive for the deposit and dissemination of scientific research documents, whether they are published or not. The documents may come from teaching and research institutions in France or abroad, or from public or private research centers.
L'archive ouverte pluridisciplinaire HAL, est destinée au dépôt et à la diffusion de documents scientifiques de niveau recherche, publiés ou non, émanant des établissements d'enseignement et de recherche français ou étrangers, des laboratoires publics ou privés. 
1 Transcriptional profiling of immune and inflammatory responses in the context of 2 SARS-CoV-2 fungal superinfection in a human airway epithelial model

4 Claire Nicolas de Lamballerie a\#, Andrés Pizzornoa\#, Julien Fouretb Lea Szpiroa, Blandine 5 Padeya,b, Julia Duboisa, Thomas Juliena,c, Aurélien Traversiera, Victoria Dulièrea,c, Pauline 6 Bruna,c, Bruno Linaa,d, Manuel Rosa-Calatravaa,c\&, Olivier Terriera\&*

8 a CIRI, Centre International de Recherche en Infectiologie, (Team VirPath), Univ Lyon, Inserm, U1111, 9 Université Claude Bernard Lyon 1, CNRS, UMR5308, ENS de Lyon, F-69007, Lyon, France.

10 ๖ Signia Therapeutics SAS, Lyon, France.

11 c VirNext, Faculté de Médecine RTH Laennec, Université Claude Bernard Lyon 1, Université de Lyon, 12 Lyon, France.

13 d Laboratoire de Virologie, Centre National de Référence des virus Influenza Sud, Institut des Agents 14 Infectieux, Groupement Hospitalier Nord, Hospices Civils de Lyon, Lyon, France.

15

16 *Correspondence to: olivier.terrier@univ-lyon1.fr (OT)

17 \# CNdL and AP are co-first authors

$18 \&$ MRC and OT are co-last authors 


\section{Abstract}

21 Superinfections of bacterial/fungal origin are known to affect the course and severity of 22 respiratory viral infections. An increasing number of evidence indicate a relatively high 23 prevalence of superinfections associated with COVID-19, including invasive aspergillosis, but 24 the underlying mechanisms remain to be characterized. In the present study, to better 25 understand the biological impact of superinfection we sought to determine and compare the 26 host transcriptional response to SARS-CoV-2 versus Aspergillus superinfection, using a model 27 of reconstituted humain airway epithelium. Our analyses reveal that both simple infection and 28 superinfection induce a strong deregulation of core components of innate immune and 29 inflammatory responses, with a stronger response to superinfection in the bronchial epithelial 30 model compared to its nasal counterpart. Our results also highlight unique transcriptional 31 footprints of SARS-CoV-2 Aspergillus superinfection, such as an imbalanced type I/type III IFN, 32 and an induction of several monocyte- and neutrophil associated chemokines, that could be 33 useful for the understanding of Aspergillus-associated COVID-19 and but also management of severe forms of aspergillosis in this specific context. 


\section{INTRODUCTION}

The current pandemic of novel coronavirus disease 2019 (COVID-19), caused by severe acute respiratory syndrome coronavirus 2 (SARS-CoV-2) began in Wuhan, Hubei province, China, in December 2019. As of May 18, 2020, there have been more than 4,628,903 confirmed COVID-19 cases in the world as reported by the WHO, including 312,009 deaths (WHO). SARS-CoV-2 is a beta-coronavirus closely related to the severe acute respiratory syndrome coronavirus-1 (SARS-CoV-1) and the Middle East respiratory syndrome coronavirus (MERSCoV) that emerged in 2003 and 2012, respectively. These viruses are also transmitted from animals to humans and cause severe respiratory diseases in afflicted individuals.

In a short period of time, significant effort has been devoted to understanding the molecular basis of the pathology associated with SARS-CoV-2 infection in an attempt to guide work on treatment, vaccine and diagnostic test development. Numerous clinical studies have reported the pathophysiology of COVID-19 has similar aspects to that initially described for SARS-CoV, i.e. acute lung injury due to over-inflammation following early stages driven by infection and viral replication (Chen et al., 2020; Guan et al., 2020; Huang et al., 2020; Mehta et al., 2020; Zhu et al., 2020). Nevertheless, the particular underlying mechanisms of these exuberant inflammatory responses in SARS-CoV-2 infection remain largely unknown and there is a need to expand our knowledge of the host's response. In this context, several recent omics-based approaches, including in vivo and in vitro transcriptional profiling studies, have highlighted specific aspects of the signature of infection that could contribute to COVID-19 (Blanco-Melo et al., 2020; Gordon et al., 2020; Messina et al., 2020; Xiong et al., 2020). Blanco-Melo and colleagues, using transcriptional and serum profiling of COVID-19 patients, have notably shown that the SARS-CoV-2 infection signature was defined by low levels of Type I and III interferons juxtaposed to elevated chemokines and high expression of IL- 6 (Blanco-Melo et al., 2020).

62

It is now well known that superinfections of bacterial/fungal origin can affect the course and severity of respiratory viral infections. For example, the co-pathogenesis of viruses and bacteria into the lung has been extensively studied, notably in the context of influenza superinfection by bacteria such as S. pneumoniae (Bosch et al., 2013; McCullers, 2014; Morens et al., 2008; Paget and Trottein, 2019). To date, there are limited data available on superinfections associated with COVID-19, though superinfections were reported in $10 \%-20 \%$ of SARS-CoV-2-infected adults admitted to Wuhan hospitals through the end of January 2020, and notably in 50\%-100\% of those who died (Zhou et al., 2020). In intensive care units, COVID- 
invasive pulmonary aspergillosis (Lescure et al., 2020). Indeed, a recent study on the French COVID-19 cohort reported that 33\% of critically ill COVID-19 patients also showed invasive aspergillosis (Alanio et al., 2020). However, while the reasons for increased vulnerability to Aspergillus in COVID-19 patients remain undetermined, the putative contribution of Aspergillus to SARS-CoV-2 related lung inflammation and COVID-19 pathophysiology also constitutes a major unanswered question.

To better understand the biological impact of superinfection in the SARS-CoV-2 context we sought to determine and compare the host transcriptional response to SARS-CoV-2 versus that of a SARS-CoV-2 + Aspergillus superinfection. To reach this goal, we established a model of SARS-CoV-2 infection and superinfection in reconstituted human airway epithelia (HAE), based on previously published works (Nicolas de Lamballerie et al., 2019; Pizzorno et al., 2019, 2020). Our analysis reveals that both simple infection and superinfection induce a strong deregulation of core components of innate immune and inflammatory responses, however, it also highlights unique transcriptional footprints of the SARS-CoV-2 + Aspergillus superinfection that provide valuable insight for the understanding not only of Aspergillusassociated COVID-19 but also for the management of severe forms of aspergillosis.

\section{RESULTS}

In order to identify similarities and differences between the host response to SARS-CoV-2 simple infection and Aspergillus superinfection, we sought to investigate the transcriptome of human respiratory epithelial cells during infection, in comparison with non-infected cells. Aware of the inherent limitations of experimental models using cell lines, we set up a model of infection/superinfection in a physiological model of reconstituted human airway epithelium (HAE). Developed from biopsies of nasal or bronchial cells differentiated in the air/liquid interphase, these HAE models, that we previously used with different respiratory viruses including SARS-CoV-2 (Nicolas de Lamballerie et al., 2019; Pizzorno et al., 2019, 2020) reproduce with high fidelity most of the main structural, functional and innate immune features of the human respiratory epithelium that play a central role during infection, hence constituting an interesting surrogate to study airway disease mechanisms. We infected nasal or bronchial HAE with SARS-CoV-2, and superinfection with Aspergillus was performed at 48 hours postinfection (hpi), which we had previously defined as the peak of acute SARS-CoV-2 infection in

106 the HAE model (Pizzorno et al., 2020). Mock-infected, infected (CoV) and superinfected 107 samples (CoV+Asp) were harvested at $72 \mathrm{hpi}$ (24 h after superinfection) to perform mRNAseq analysis (Fig. 1A). Both nasal and bronchial HAE models of superinfection were further 
109 characterized and validated in terms of viral production, impact on trans-epithelial resistance

110 and apical release of IL-6, which we used as hallmarks of infection (Fig. 2A). Interestingly, in 111 contrast with nasal HAE, we observed a significative increase on the relative viral production

112 in the context of superinfection in bronchial HAE, associated with higher IL-6 levels and a 113 stronger negative impact on trans-epithelial resistance (Fig. 1B).

115 Differential expression analysis of mRNA-seq data compared to the mock-infected condition identified 1638 and 454 differentially expressed genes (DEGs) in SARS-CoV-2-infected nasal and bronchial HAE, respectively ( $F C \geq 2$, Benjamini-Hochberg adjusted $p$-value $<0.01$ ). In the context of $\mathrm{CoV}+\mathrm{Asp}$ superinfection, the number of DEGs was notably higher, with 2979 and 3235 genes in nasal and bronchial HAE, respectively (Extended data. File 1). Interestingly,

120 there was an important overlap between CoV infection and CoV+Asp superinfection

121 associated DEGs in both nasal and bronchial models. As illustrated in the Venn diagrams (Fig.

122 1C), more than 96\% (nasal HAE) and 99\% (bronchial HAE) of DEGs of the CoV signature 123 were also part of the $\mathrm{CoV}_{+}$Asp signature. To provide further functional interpretation of the 124 global transcriptional signatures, we performed a functional enrichment analysis on the CoV 125 and CoV+Asp nasal and bronchial models using the web-based DAVID toolkit. Gene Ontology 126 (GO), UniProt $(\mathrm{KW})$ and Reactome (RC) terms where considered enriched when their 127 Bonferroni-adjusted corrected enrichment $p$-value was < 0.01 (Fig. 1D). As anticipated, a large 128 part of the most enriched and shared terms between all experimental conditions was related 129 to the host response to infection (Regulation of response to cytokine stimulus, regulation of 130 defense response, regulation of response to biotic stimulus) and also to cornification, which 131 regroups genes mostly involved in cell-death mechanisms (Fig. 1D). Interestingly, functional 132 enrichments specific to $\mathrm{CoV}$ infection or $\mathrm{CoV}+$ Asp superinfection were also highlighted. For 133 example, CoV infection but no superinfection induced the upregulation of a gene cluster 134 involved in Type I interferon pathway (Fig. 1D). Conversely, gene clusters harboring terms 135 associated with epithelium physiology and cilium movements (regulation of cellular component 136 movements, cell projection assembly, intraflagellar transport) but also with intra and extra137 cellular signaling (post-translational protein phosphorylation, protein localization to 138 extracellular membrane, peptide secretion) were exclusively enriched in the context of 139 CoV+Asp superinfection (Fig. 1D). In parallel, given the importance of the exacerbated 140 immune response observed mainly in severe COVID-19 cases, we further analyzed the 141 inflammation-related terms and identified similar, yet to a different extent, regulation patterns 142 for genes involved in the positive regulation of inflammatory response (GO:0050729) between 143 nasal and bronchial HAE. As exemplified in the heatmap (Fig. 1D), deregulated genes such 144 as calgranulin coding genes S100A12 and S100A9/A8 and downregulated genes such as 145 Vesicle-associated membrane protein coding genes VAMP7 or VAMP8 were identified as a 
146 hallmark of $\mathrm{CoV}+$ Asp superinfection, in contrast to CoV infection (Fig. 1D). Similar 147 observations were performed using different terms related to inflammation (Extended data

148 Fig. 1), hence suggesting a very different inflammation signature resulting from superinfection.

149 Altogether, our results indicate that the CoV+Asp superinfection presents a transcriptomic 150 signature that recapitulates the overall signature of a simple CoV infection, but with both a 151 particularly distinct regulation of the inflammatory response and the additional regulation of 152 many biological processes related to the physiology of epithelia. Of note, we observed a 153 relatively similar global pattern of regulation between the nasal and bronchial HAE models, 154 differing primarily in the magnitude rather than the nature of the responses to CoV and 155 CoV+Asp superinfection.

157 In order to explore in more depth the transcriptomic signature of the superinfection, we then 158 performed a differential analysis of $\mathrm{CoV}+\mathrm{Asp}$ signature using that of CoV infection as baseline.

159 This focused analysis highlighted 248 and 769DEGs in the CoV+Asp nasal and bronchial HAE, 160 respectively $(F C \geq 2$, adjusted $p$-value $<0.01$ ), which allowed us to spot finer differences between the two epithelium models. Indeed, the volcano plots in Fig. 2A and B representing all DEGs induced by the CoV+Asp versus the CoV condition show interesting differences in both the scale of deregulation and the nature of the most deregulated genes between the upper and lower respiratory tract tissues. Interestingly, many genes involved in the regulation of the inflammatory response such as HOX1, IL1B, IL1A, IL17C are found amongst the most upregulated genes in the nasal HAE model (Fig. 2A). These genes are also upregulated in the bronchial HAE model (Extended data file 2). To provide further functional interpretation of the superinfection signature, we performed a functional enrichment analysis, using the same strategy previously described. We only observed a limited number of enriched clusters of downregulated genes, mostly in bronchial HAE, all of them being related to epithelial physiology and cell movement (e.g. cytoskeleton-dependent intracellular transport, protein localization to cilium, cilium movement GO terms, Fig. 2C). In line with this observation, our

173 analysis highlighted several clusters of enriched upregulated genes related to epithelial 174 physiology (e.g. locomotion, movement of cell or subcellular component GO terms, Fig. 2C).

175 On the other hand, terms related to signaling, host response and immunity were markedly 176 more upregulated in the bronchial HAE model (Fig. 2C \& Extended data Fig. 2). In addition 177 to the gene enrichment profile shared between the nasal and bronchial models, our study also 178 highlighted several overlapping biological processes such as the cytokine signaling and IL10 179 signaling pathways (Fig. 2C), which is consistent with the most upregulated DEGs shown in 180 Fig. 2A and Extended data file 2. To better visualize these observations, we applied a protein181 protein interactions analysis using STRING network to investigate the DEGs corresponding to 182 several Reactome and GO terms (immune system process, cytokine signaling in immune 
system, inflammatory response, interleukin-10 signaling) enriched in the bronchial and nasal superinfection signatures along with their functional interactions (Fig. 3A and 3B). The two interactome maps with mostly upregulated DEGs illustrate the strong functional interconnections among several cytokines/chemokines (blue) and receptors (yellow) involved in the immune and inflammatory responses occurring in the context of a SARS-CoV-2 + Aspergillus superinfection. Interestingly, our analysis underlined the role of type III IFN (IFNL1, INFL2 and INFL3) and several cytokine-coding genes such as CXCL2/CXCL8 (Fig. 3A and 3B) that are upregulated following simple SARS-CoV-2 infection and even more upregulated in the context of superinfection, arguably illustrating an enhanced specific response to control infection in both models.

\section{DISCUSSION}

Invasive pulmonary aspergillosis (IPA), which typically occurs in an immunocompromised host, represents an important cause of morbidity and mortality worldwide (Clancy and Nguyen, 2020). Superinfections were extensively documented in the case of influenza infections, with the latter being usually described to "pave the way" for bacterial superinfections, but several severe influenza cases have also been reported to develop invasive pulmonary aspergillosis 5/19/2020 9:59:00 AM. An increasing amount of evidence points towards a relatively high prevalence of superinfections, including invasive aspergillosis, to be associated with COVID19 (Alanio et al., 2020; Lescure et al., 2020; Zhou et al., 2020). However, the underlying mechanisms remain to be characterized. In the present study, we sought to better understand the biological impact of superinfections by determining and comparing the host transcriptional response to SARS-CoV-2 versus SARS-CoV-2 + Aspergillus superinfection. Collectively, our results show a much stronger host response to superinfection in the bronchial epithelial model compared to its nasal counterpart. In both models, functional analyses show that the SARSCoV-2 + Aspergillus superinfection signature reflects important changes in the expression regulation of genes involved not only in epithelium physiology but also in the regulation of host immune and inflammatory responses compared to that of the simple SARS-CoV-2 infection.

The reconstituted HAE model of infection/superinfection appears as a valuable support for the study of respiratory viral infections and virus-host interactions in highly biologically relevant experimental conditions. Previous results by our group using this model, constituted of fully differentiated and functional human primary cells, have provided meaningful contributions to the characterization of the kinetics of viral infection as well as on the tissue-level remodeling of the cellular ultrastructure and local innate immune responses induced by SARS-CoV-2, in 
220 line with our present observation (Pizzorno et al., 2020). Whereas no major differences in 221 terms of global superinfection signatures where observed between HAE models of nasal or 222 bronchial origin, the second part of our study highlighted more subtle differences between the 223 two models in terms of scale of deregulation (fold change and p-value), as well as in the nature 224 of the most deregulated genes (Fig. 2C). Ziegler and colleagues have recently reported that differences of infectivity and consecutive host responses between different cell subsets (type II pneumocytes, nasal goblet secretory cells) are linked to varying ACE2/TMPRSS2 levels, ACE2 expression being linked to the IFN response (Ziegler et al., 2020). The discrepancies we observed in the two HAE models could be explained by differences of cell type composition that could be interesting to further explore using combinations of additional experimental models, including ACE2/TMPRSS3 expression and single cell RNA-seq approaches.

Our analysis of the superinfection signature revealed an important role of physiology and cilium-related genes, which could reflect an additional negative impact of Aspergillus infection on the epithelium integrity (trans-epithelial resistance, Fig. 1B) and mucociliary clearance, in good agreement with previous observations by our group following different types of viral infection in HAE (Nicolas de Lamballerie et al., 2019). In the specific context of SARS-CoV-2 infection, the additional deleterious effect on epithelium integrity induced by aspergillosis might contribute to the enhanced disease severity reported in the clinic while arguably increasing the risk of additional bacterial of fungal superinfections, similarly to what has been described in the case of other respiratory viral infections (Smith et al., 2013; Wu et al., 2016).

Another major finding of our study relates to the impact of infection/superinfections on interferon and inflammatory responses, notably when compared to the limited transcriptomic data available in literature to date. Using primary cells, Blanco-Melo and colleagues recently reported that SARS-CoV-2 induces limited IFN-I and III responses (Blanco-Melo et al., 2020). Our data are not entirely consistent with these findings. Whereas we also demonstrate a very limited involvement of the type I interferon response in the context of simple SARS-CoV-2 infection -in contrast with data previously obtained with other viruses (Nicolas de Lamballerie et al., 2019), our analysis highlights an important upregulation of crucial genes involved in the type IIII interferon response. This response is even more pronounced in the context of superinfection (Fig. 3A and 3B). Type III interferon (IFN- $\lambda$ ) is known to play a key role in innate and adaptive mucosal immune responses to infection (Ye et al., 2019). Interestingly, IFN- $\lambda$ has been identified as a critical regulator of neutrophil activation to prevent fungal infection such as invasive pulmonary aspergillosis (Espinosa et al., 2017). Our data suggest a divergence between type I and type III interferon responses, with enhanced activation of the latter in the context of fungal superinfection. This characteristic response could be associated with 
257 increased neutrophil activation as an important first line of adaptive defense against these two 258 pathogens. This hypothesis is further supported by the observed induction of several 259 monocyte-and neutrophil associated chemokines, such as CCL2, CXCL2 and CXCL3 (Fig. 3A 260 and $\mathbf{B}$ ). Interestingly, our results suggest a strong activation of adaptive immune response in 261 the context of SARS-CoV-2 infection, in line with currently available clinical data from COVID26219 patients, which generally present high levels of circulating neutrophils (Chen et al., 2020; 263 Qin et al., 2020). These observations support an important role of neutrophil recruitment in the 264 response to COVID-19, more particularly in the context of fungal superinfection with an 265 exacerbation of pro-inflammatory response. In that sense, the upregulation of key genes 266 belonging to the IL-10 pathway observed in our analysis (Fig. 2C), previously demonstrated to 267 play a deleterious role in innate resistance to systemic aspergillosis (Clemons et al., 2000), 268 constitutes an illustration of how such "enhanced" state of inflammation could contribute to 269 increase severity.

271 In conclusion, our transcriptional profiling approach revealed unique features of the SARS272 CoV-2 + Aspergillus superinfection signature, characterized on one side by an "enhanced" 273 version of that induced by SARS-CoV infection, but also by specific changes on respiratory 274 tissue physiology, a distinct regulation of type I and type III interferons, and an over-induction 275 of inflammatory response. While we acknowledge that it would be rather bold to make a 276 statement on the possible severity associated with Aspergillus superinfection in a pre-existing 277 COVID-19 pathological context based solely on our results, our observations suggest that the 278 immunomodulation induced by SARS-CoV-2 infection could establish a favorable context for 279 the development of severe forms of aspergillosis. This could constitute an important aspect to 280 be considered for the immunological follow-up of COVID-19 patients with aspergillosis. On top 281 of that, the characteristic infection signatures described in this study provide valuable insight 282 in the perspective of possible future treatments targeting the COVID-19 inflammatory response, 283 which could result in counter-productive effects for the management of aspergillosis. 
MATERIALS AND METHODS

\section{Reconstituted human airway epithelial model}

287 MucilAirtm HAE reconstituted from human primary cells obtained from nasal or bronchial 288 biopsies, were provided by Epithelix SARL (Geneva, Switzerland) and maintained in air-liquid interphase with specific culture medium in Costar Transwell inserts (Corning, NY, USA) according to the manufacturer's instructions. For infection experiments, apical poles were gently washed twice with warm OptiMEM medium (Gibco, ThermoFisher Scientific) and then infected directly with $150 \mu$ dilution of virus in OptiMEM medium, at a multiplicity of infection (MOI) of 0.1 (Pizzorno et al., 2020). For mock infection, the same procedure was followed using OptiMEM as inoculum. Superinfection was performed in SARS-CoV-2 infected cells at 48h post-infection, with apical inoculation of $10 \mu \mathrm{l}$ of Aspergillus in OptiMEM at a MOI of 1 . Samples were collected from apical washes or basolateral medium at $72 \mathrm{~h}$ post infection. Variations in transepithelial electrical resistance (TEER) were measured using a dedicated volt-ohm meter (EVOM2, Epithelial Volt/Ohm Meter for TEER) and expressed as Ohm/cm2.

\section{Pathogens}

All experiments involving clinical samples and the manipulation of infectious SARS-CoV-2 were performed in biosafety level 3 (BSL-3) facilities, using appropriate protocols. The BetaCoV/France/IDF0571/2020 SARS-CoV-2 strain used in this study was isolated directly from a patient sample as described elsewhere (Pizzorno et al., 2020). Viral stocks were prepared and quantified in Vero E6 cells (TCID50/mL). Aspergillus niger (ATCC 16404) was quantified on maltose extract agar plates (CFU/ml).

\section{mRNA sequencing}

309 Total RNA was extracted using the RNeasy Mini Kit (QIAGEN, ref 74104) with DNase 310 treatment, following the manufacturer's instruction. $500 \mu \mathrm{g}$ total RNA was used to prepare 311 polyA-enriched RNA-seq libraries using the KAPA mRNA Hyper Prep Kit (Roche, ref. 312 KK8581/KK8581). Those libraries were prepared separately for each sample with 11 313 amplification cycles, then all libraries were equimolar-pooled before sequencing. Paired-end 314 sequencing $(2 \times 100 \mathrm{bp})$ was performed with Illumina NovaSeq 6000 sequencing platform on a 315 SP flowcell (Illumina, ref: 20040326).

\section{RNA-Seq data trimming}

318 Raw reads were first cleaned with Cutadapt 2.8 (Martin, 2011) to trim adapters 
reads) and low-quality ends (i.e terminal bases with phred quality score below 30). Only reads longer than $75 \mathrm{bp}$ and with less than $20 \%$ of $\mathrm{N}$ (undetermined base) after trimming were kept

323 for further analysis.

\section{Transcript abundance quantification}

326 The Kallisto 0.46 .1 software was used for reference-indexing and transcript abundance estimation (Bray et al., 2016). The reference transcriptome based on NCBI RefSeq annotation release 109.20191205 and genome assembly build GRCh37.p13 were chosen for this analysis (Maglott et al., 2005). Default options were used for Kallisto except for reads orientation that

330 was specified. The Extended Data Table 2 provides a summary statistics of the pseudoalignment process.

\section{Differential expression analysis}

334 Differential expression analysis was performed with R 3.6.3. First abundances at transcriptlevel were imported using tximport 1.14.0 (Soneson et al., 2015). Only coding mRNA transcripts were considered. Gene-level effective lengths were obtained by weighted mean with their expression values in FPKM (Fragment Per Kilobase Million) weights. Raw counts were also computed at gene-level by sum and used as input for DESeq2 1.26.0 (Love et al., 2014). Normalization within DESeq2 used first gene-level effective length and then the size factor that was estimated using the function 'estimateSizeFactors'. Differential expression testing was performed using default parameters. P-values were adjusted using the BenjaminiHochberg method (Benjamini and Hochberg, 1995) after the independent filtering implemented by default in DESeq2. A gene was considered differentially expressed if both the adjusted $p$ value is below 0.01 and the induced change in expression is at least a two-fold increase (for up-regulated genes) or a two-fold decrease (for down-regulated genes). Finally, the NCBI gene IDs were mapped to Uniprot IDs using Uniprot cross-references (https://www.uniprot.org/database/DB-0118) (Breuza et al., 2016; Maglott et al., 2005). First, Uniprot entries associated to several gene IDs were removed. Indeed, we are not focused on gene fusion products or read-through transcripts in this study. In case of a gene ID associated to several Uniprot entries, only the best entry based on the reviewed status and the annotation score was kept; if those values were not discriminant enough, only duplicated entries with a reviewed status and an annotation score of 5 were kept. The protein-protein interaction (PPI) network was analyzed with STRING 11.0 and visualized with Cytoscape 3.8.0.

\section{In silico functional analysis}

356 Based on mapped Uniprot IDs, gene set enrichment was performed using the parent-child 357 union method as previously described (Grossmann et al., 2007). In this method each functional 
358 term, called child, is considered relatively to its parents to compute the probability of 359 enrichment ( $p$-value). We considered three functional databases with parent-child relations: 360 Reactome (Fabregat et al., 2018), Uniprot Keyword (restricted to "Biological process" and 361 "Disease") (Breuza et al., 2016) and Gene ontology (Restricted to "Biological process")

362 (Ashburner et al., 2000; The Gene Ontology Consortium, 2015). Different files (available online: 363 URLs were all accessed the 04/02/2020) were parsed to define Uniprot ID associations with 364 terms and identify child to parents relations within each functional database; URLs are 365 specified in Extended Data Table 1. As previously defined (Grossmann et al., 2007), the 366 p_min probability represents how well a child term can be enriched relative to its parents. The 367 higher this probability is, the less informative is the child term relative to its parents. This is 368 also a statistic that can be used for independent filtering to reduce the $p$-value adjustment 369 burden. Therefore, all terms with a p_min probability higher than $10 \mathrm{e}-4$ were filtered out before 370 p-values adjustment using Bonferroni method (Dunn, 1961). This multiple-testing correction 371 procedure was handled separately for each functional database and analyzed gene list. A 372 minimum adjusted $p$-value of 0.05 has been set for significant enrichment.

\section{Acknowledgments}

375 The authors would like to thank Epithelix (Switzerland) and IGENSEQ sequencing core facility 376 (Institut du Cerveau ICM, Paris) for their help. This study was funded by CNRS, and Mérieux 377 research grants. The sponsors had no role in study design, collection, analysis and 378 interpretation of data, manuscript writing, or in the decision to submit the article for publication. 


\section{REFERENCES}

Alanio, A., Delliere, S., Fodil, S., Bretagne, S., and Megarbane, B. (2020). High prevalence of putative invasive pulmonary aspergillosis in critically ill COVID-19 patients. MedRxiv 2020.04.21.20064915.

Blanco-Melo, D., Nilsson-Payant, B.E., Liu, W.-C., Uhl, S., Hoagland, D., Møller, R., Jordan, T.X., Oishi, K., Panis, M., Sachs, D., et al. (2020). Imbalanced Host Response to SARS-CoV2 Drives Development of COVID-19. Cell.

Bosch, A.A.T.M., Biesbroek, G., Trzcinski, K., Sanders, E.A.M., and Bogaert, D. (2013). Viral and Bacterial Interactions in the Upper Respiratory Tract. PLOS Pathog 9, e1003057.

Bray, N.L., Pimentel, H., Melsted, P., and Pachter, L. (2016). Near-optimal probabilistic RNAseq quantification. Nat. Biotechnol. 34, 525-527.

Breuza, L., Poux, S., Estreicher, A., Famiglietti, M.L., Magrane, M., Tognolli, M., Bridge, A., Baratin, D., Redaschi, N., and UniProt Consortium (2016). The UniProtKB guide to the human proteome. Database J. Biol. Databases Curation 2016.

Chen, N., Zhou, M., Dong, X., Qu, J., Gong, F., Han, Y., Qiu, Y., Wang, J., Liu, Y., Wei, Y., et al. (2020). Epidemiological and clinical characteristics of 99 cases of 2019 novel coronavirus pneumonia in Wuhan, China: a descriptive study. The Lancet 395, 507-513.

Clancy, C.J., and Nguyen, M.H. (2020). COVID-19, superinfections and antimicrobial development: What can we expect? Clin. Infect. Dis. Off. Publ. Infect. Dis. Soc. Am.

Clemons, K.V., Grunig, G., Sobel, R.A., Mirels, L.F., Rennick, D.M., and Stevens, D.A. (2000). Role of IL-10 in invasive aspergillosis: increased resistance of IL-10 gene knockout mice to lethal systemic aspergillosis. Clin. Exp. Immunol. 122, 186-191.

Espinosa, V., Dutta, O., McElrath, C., Du, P., Chang, Y.-J., Cicciarelli, B., Pitler, A., Whitehead, I., Obar, J.J., Durbin, J.E., et al. (2017). Type III interferon is a critical regulator of innate antifungal immunity. Sci. Immunol. 2.

406 Gordon, D.E., Jang, G.M., Bouhaddou, M., Xu, J., Obernier, K., White, K.M., O’Meara, M.J., 407 Rezelj, V.V., Guo, J.Z., Swaney, D.L., et al. (2020). A SARS-CoV-2 protein interaction map 408 reveals targets for drug repurposing. Nature 1-13.

Grossmann, S., Bauer, S., Robinson, P.N., and Vingron, M. (2007). Improved detection of overrepresentation of Gene-Ontology annotations with parent child analysis. Bioinforma. Oxf. Engl. 23, 3024-3031.

412 Guan, W., Ni, Z., Hu, Y., Liang, W., Ou, C., He, J., Liu, L., Shan, H., Lei, C., Hui, D.S.C., et al. (2020). Clinical Characteristics of Coronavirus Disease 2019 in China. N. Engl. J. Med. 382, 414 1708-1720. 
Lescure, F.-X., Bouadma, L., Nguyen, D., Parisey, M., Wicky, P.-H., Behillil, S., Gaymard, A., Bouscambert-Duchamp, M., Donati, F., Le Hingrat, Q., et al. (2020). Clinical and virological data of the first cases of COVID-19 in Europe: a case series. Lancet Infect. Dis.

Love, M.I., Huber, W., and Anders, S. (2014). Moderated estimation of fold change and dispersion for RNA-seq data with DESeq2. Genome Biol. 15, 550.

Maglott, D., Ostell, J., Pruitt, K.D., and Tatusova, T. (2005). Entrez Gene: gene-centered information at NCBI. Nucleic Acids Res. 33, D54-D58.

Martin, M. (2011). Cutadapt removes adapter sequences from high-throughput sequencing reads. EMBnet.Journal 17, 10-12.

McCullers, J.A. (2014). The co-pathogenesis of influenza viruses with bacteria in the lung. Nat. Rev. Microbiol. 12, 252-262.

Mehta, P., McAuley, D.F., Brown, M., Sanchez, E., Tattersall, R.S., and Manson, J.J. (2020). COVID-19: consider cytokine storm syndromes and immunosuppression. The Lancet 395, 1033-1034.

Messina, F., Giombini, E., Agrati, C., Vairo, F., Bartoli, T.A., Moghazi, S.A., Piacentini, M., Locatelli, F., Kobinger, G., Maeurer, M., et al. (2020). COVID-19: Viral-host interactome analyzed by network based-approach model to study pathogenesis of SARS-CoV-2 infection. BioRxiv 2020.05.07.082487.

Morens, D.M., Taubenberger, J.K., and Fauci, A.S. (2008). Predominant role of bacterial pneumonia as a cause of death in pandemic influenza: implications for pandemic influenza preparedness. J. Infect. Dis. 198, 962-970.

Nicolas de Lamballerie, C., Pizzorno, A., Dubois, J., Julien, T., Padey, B., Bouveret, M., Traversier, A., Legras-Lachuer, C., Lina, B., Boivin, G., et al. (2019). Characterization of cellular transcriptomic signatures induced by different respiratory viruses in human reconstituted airway epithelia. Sci. Rep. 9, 11493.

443 Paget, C., and Trottein, F. (2019). Mechanisms of Bacterial Superinfection Post-influenza: A 444 Role for Unconventional T Cells. Front. Immunol. 10.

445 Pizzorno, A., Terrier, O., Nicolas de Lamballerie, C., Julien, T., Padey, B., Traversier, A., 446 Roche, M., Hamelin, M.-E., Rhéaume, C., Croze, S., et al. (2019). Repurposing of Drugs as Novel Influenza Inhibitors From Clinical Gene Expression Infection Signatures. Front. Immunol. 10.

Pizzorno, A., Padey, B., Julien, T., Trouillet-Assant, S., Traversier, A., Errazuriz-Cerda, E., 450 Fouret, J., Dubois, J., Gaymard, A., Lescure, F.-X., et al. (2020). Characterization and 451 treatment of SARS-CoV-2 in nasal and bronchial human airway epithelia. BioRxiv $452 \quad 2020.03 .31 .017889$.

Qin, C., Zhou, L., Hu, Z., Zhang, S., Yang, S., Tao, Y., Xie, C., Ma, K., Shang, K., Wang, W., et al. (2020). Dysregulation of immune response in patients with COVID-19 in Wuhan, China. Clin. Infect. Dis. Off. Publ. Infect. Dis. Soc. Am. 
459 Soneson, C., Love, M.I., and Robinson, M.D. (2015). Differential analyses for RNA-seq:

460 transcript-level estimates improve gene-level inferences. F1000Research 4, 1521.

461 Wu, N.-H., Yang, W., Beineke, A., Dijkman, R., Matrosovich, M., Baumgärtner, W., Thiel, V., 462 Valentin-Weigand, P., Meng, F., and Herrler, G. (2016). The differentiated airway epithelium 463 infected by influenza viruses maintains the barrier function despite a dramatic loss of ciliated 464 cells. Sci. Rep. 6, 39668.

465 Xiong, Y., Liu, Y., Cao, L., Wang, D., Guo, M., Jiang, A., Guo, D., Hu, W., Yang, J., Tang, Z., 466 et al. (2020). Transcriptomic characteristics of bronchoalveolar lavage fluid and peripheral 467 blood mononuclear cells in COVID-19 patients. Emerg. Microbes Infect. 9, 761-770.

468 Ye, L., Schnepf, D., and Staeheli, P. (2019). Interferon- $\lambda$ orchestrates innate and adaptive 469 mucosal immune responses. Nat. Rev. Immunol. 19, 614-625.

470 Zhou, F., Yu, T., Du, R., Fan, G., Liu, Y., Liu, Z., Xiang, J., Wang, Y., Song, B., Gu, X., et al. (2020). Clinical course and risk factors for mortality of adult inpatients with COVID-19 in Wuhan, China: a retrospective cohort study. Lancet Lond. Engl. 395, 1054-1062.

Zhu, N., Zhang, D., Wang, W., Li, X., Yang, B., Song, J., Zhao, X., Huang, B., Shi, W., Lu, R., et al. (2020). A Novel Coronavirus from Patients with Pneumonia in China, 2019. N. Engl. J. Med. 382, 727-733.

Ziegler, C.G.K., Allon, S.J., Nyquist, S.K., Mbano, I.M., Miao, V.N., Tzouanas, C.N., Cao, Y., Yousif, A.S., Bals, J., Hauser, B.M., et al. (2020). SARS-CoV-2 Receptor ACE2 Is an 478 Interferon-Stimulated Gene in Human Airway Epithelial Cells and Is Detected in Specific Cell Subsets across Tissues. Cell. 


\section{FIGURES}

A

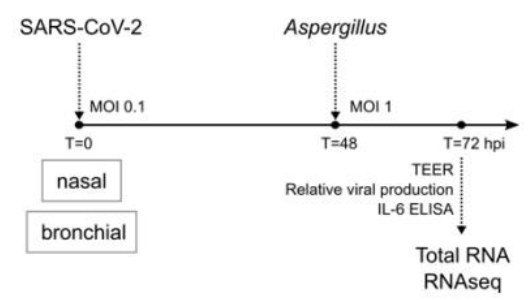

C

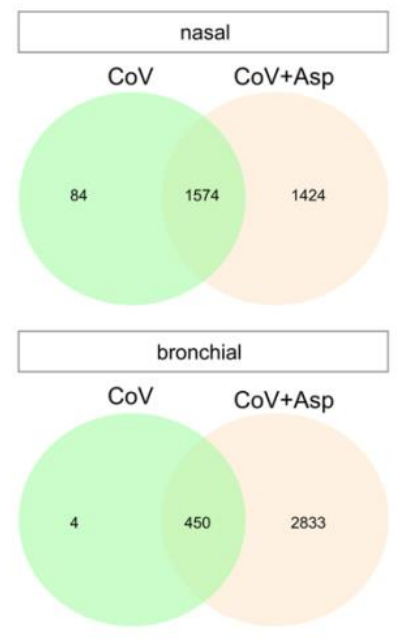

B
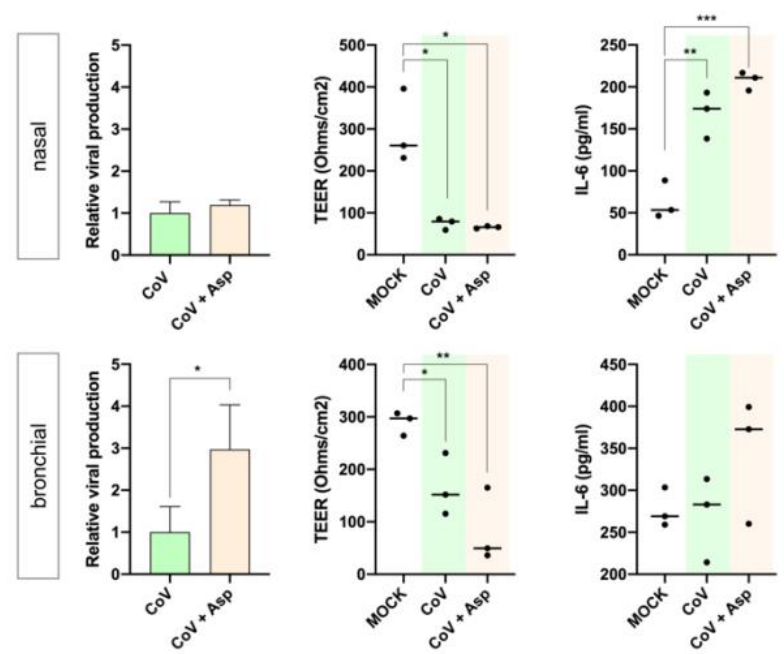

D

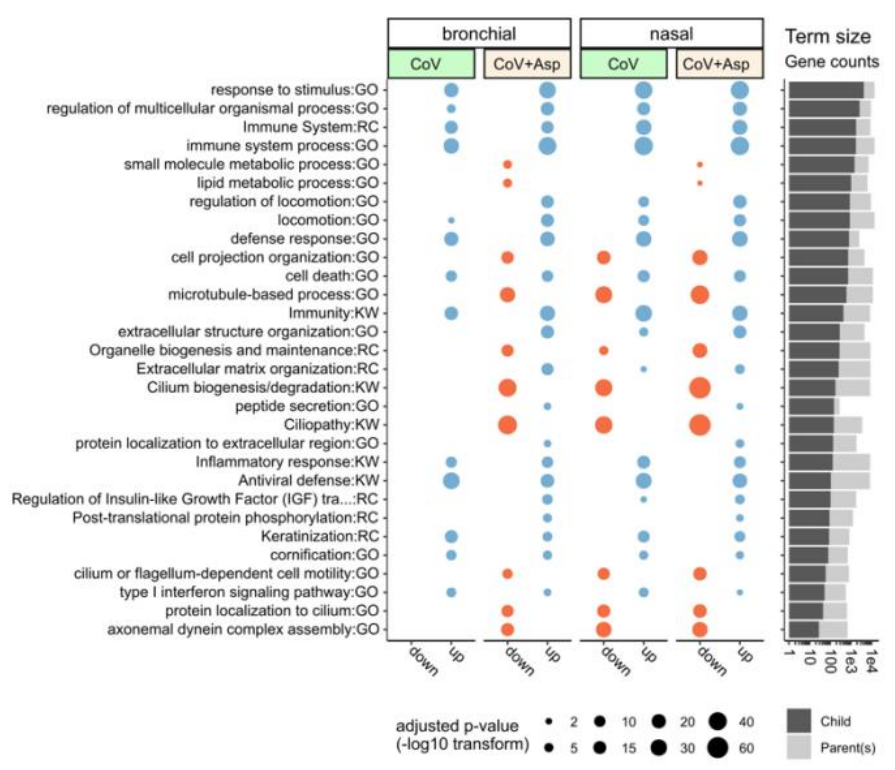

$E$

GO:0050729 - positive regulation of inflammatory response
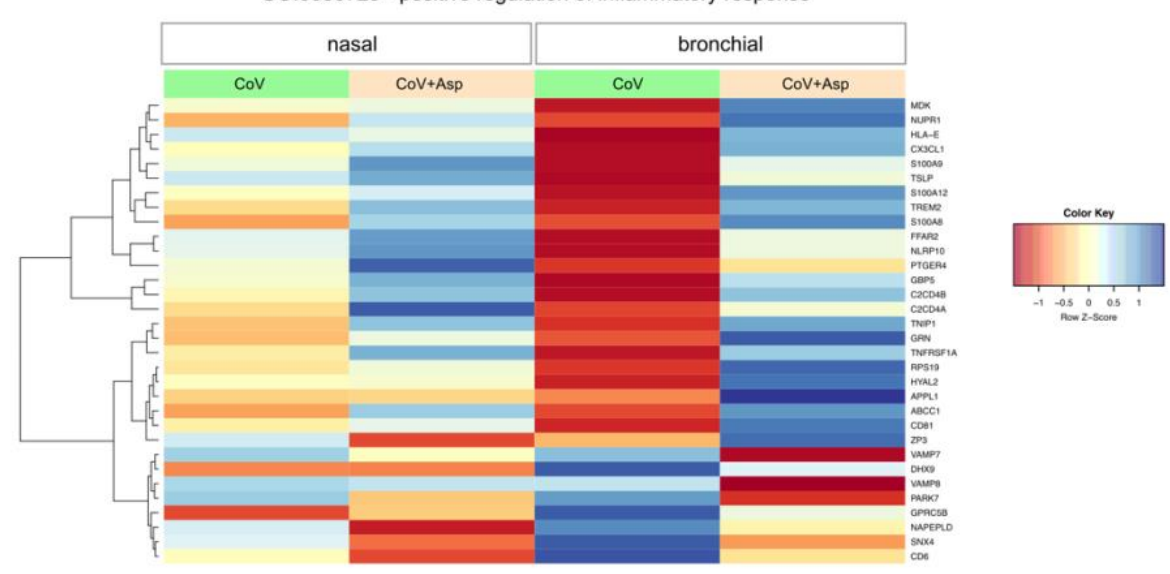


\section{Figure 1}

485 (A) Overview of experimental strategy (B) At 72h post-infection, for both nasal and bronchial 486 HAE model, the relative viral production (intracellular) was determined using RTqPCR, and 487 the impact of infection on epithelium integrity was monitored by measure of the transepithelial 488 resistance (TERR Ohms/cm2). IL-6 was measured at the apical using a specific ALISA assay. 489 (C) Nasal and bronchial gene signature overlap for SARS-CoV2 or SARS-CoV-2+Aspergillus 490 infected conditions vs. Mock. We contrasted the significantly differentially expressed gene lists 491 corresponding to CoV vs. Mock and CoV+Asp vs. Mock in nasal and bronchial HAE. Only 492 genes above threshold $(\log 2(\mathrm{FC})>1$ or $<-1$ compared to the mock-infected condition and 493 Benjamini-Hochberg adjusted $p$ value $<0.01$ ) were considered. (D) Overview of functional 494 enrichment results for SARS-CoV2 or SARS-CoV-2+Aspergillus infected conditions vs. Mock. 495 Considering (CoV vs. Mock) and (CoV+Asp vs. Mock) for both bronchial and nasal HAE, 496 significantly up- or down- regulated gene lists ( $\mathrm{x}$-axis) were tested for significant enrichment 497 using the parent-child strategy (see methods). If below the threshold $(0.01)$, the adjusted $p$ 498 values corresponding to different terms ( $y$-axis) are represented by point sizes (see legend). 499 After clustering terms based on gene occurrences (binary distance \& Ward algorithm) in 15 500 metagroups, only the top 2 (lowest adjusted $p$-value) were represented here. For the complete 501 list of significant terms, see supplementary figure $\mathrm{S} 1$. The bar plot on the right represents the 502 sizes of enriched terms (called child) in comparison to the size of their parents (see methods 503 for definitions). (E) Positive regulation of the inflammatory response in nasal and bronchial 504 HAE. We extracted the list of proteins associated with the term GO:0050729 and visualized 505 the scaled log transformed expression results in a heatmap. Each row represents a gene and 506 each column is an experimental condition. The color key indicates the scaled expression levels vs. Mock (red, low; blue, high). 
bioRxiv preprint doi: https://doi.org/10.1101/2020.05.19.103630; this version posted May 19, 2020. The copyright holder for this preprint (which was not certified by peer review) is the author/funder. All rights reserved. No reuse allowed without permission.
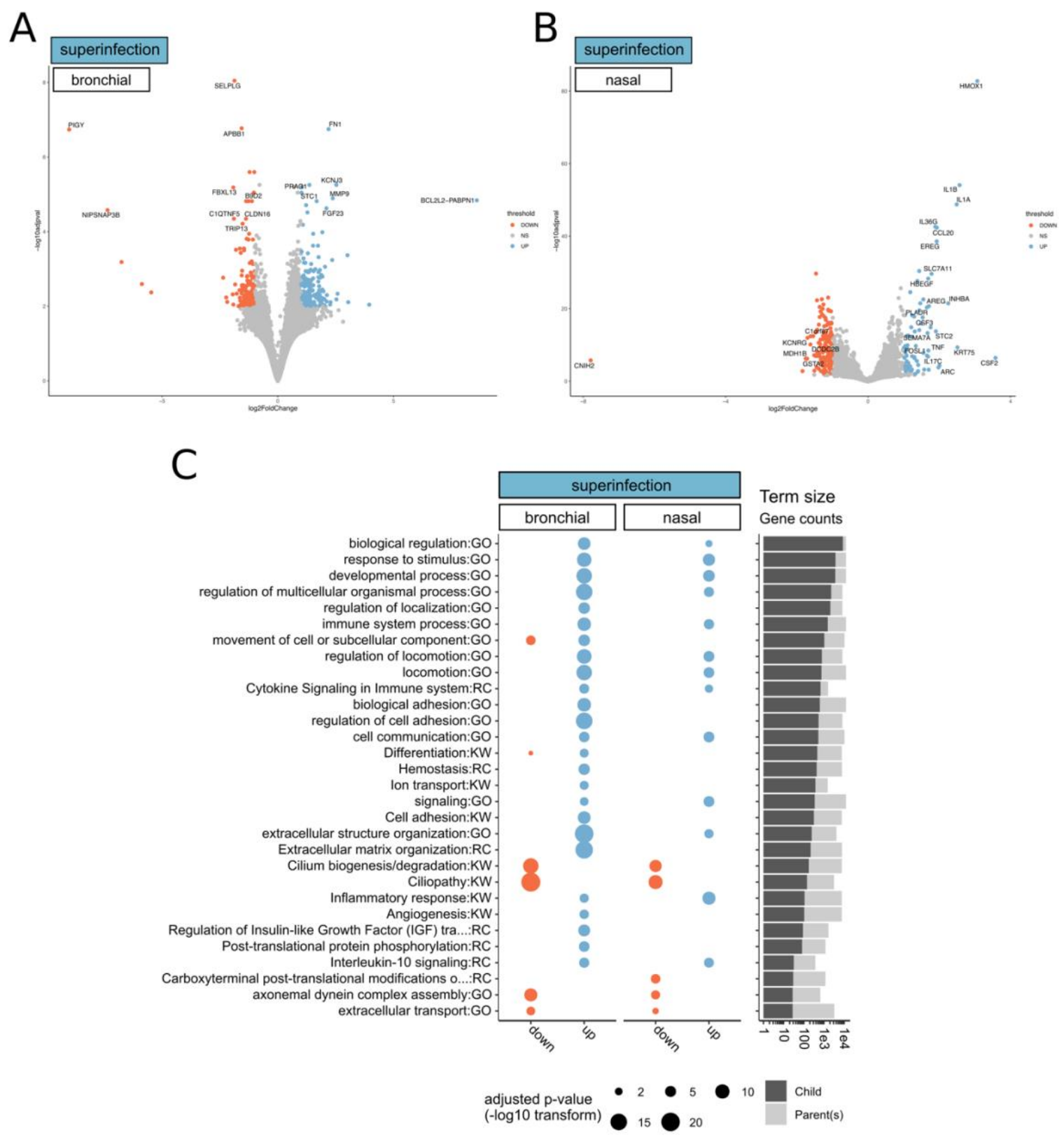


\section{Figure 2}

510 (A) \& (B) Volcano plots of differentially expressed genes from (A) nasal and (B) bronchial tissue

511 superinfected by Aspergillus. We considered significantly modulated genes (CoV+Asp vs. CoV)

512 for both bronchial and nasal HAE ( $\log 2(\mathrm{FC})>1$ or $<-1$ compared to the CoV condition and

513 Benjamini-Hochberg adjusted $p$-value $<0.01)$. The color scale represents the log-transformed

514 fold change values and ranges from low (red) to high (blue). (C) Overview of functional

515 enrichment results of SARS-CoV-2+Aspergillus superinfection vs. SARS-CoV2 infection.

516 Considering (CoV+Asp vs. CoV) for both bronchial and nasal epithelium type, significantly up-

517 or down- regulated gene lists (x-axis) were tested for significant enrichment using the parent-

518 child strategy (see methods). If below the threshold (0.01), the adjusted p-values

519 corresponding to different terms (y-axis) are represented by point sizes (see legend). After

520 clustering terms based on gene occurrences (binary distance \& Ward algorithm) in 15

521 metagroups, the top 2 in terms lowest $p$-value were represented here. For the complete list of

522 significant terms, see Extended Data File 2. The bar plot on the right represents the sizes of

523 enriched terms (called child) in comparison to the size of their parents (see methods for

524 definitions). 
bioRxiv preprint doi: https://doi.org/10.1101/2020.05.19.103630; this version posted May 19, 2020. The copyright holder for this preprint (which was not certified by peer review) is the author/funder. All rights reserved. No reuse allowed without permission.

\section{A}

superinfection

bronchial

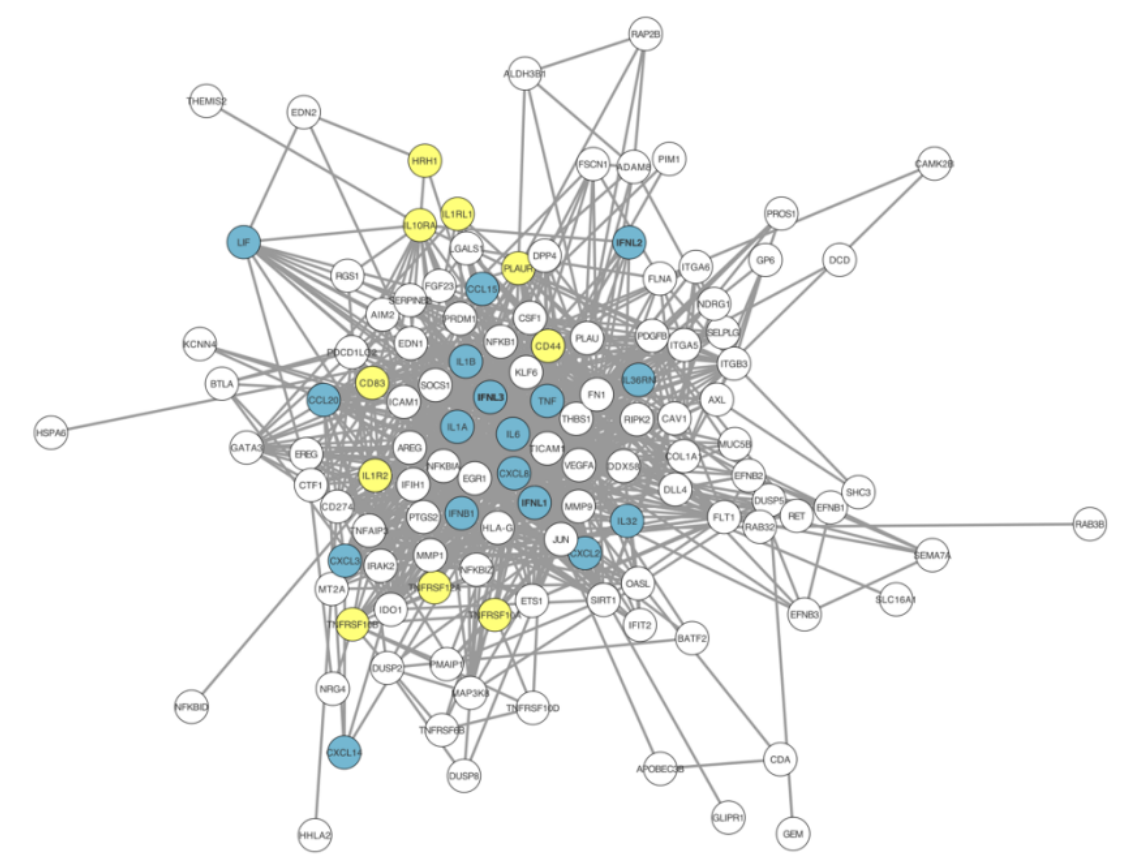

B

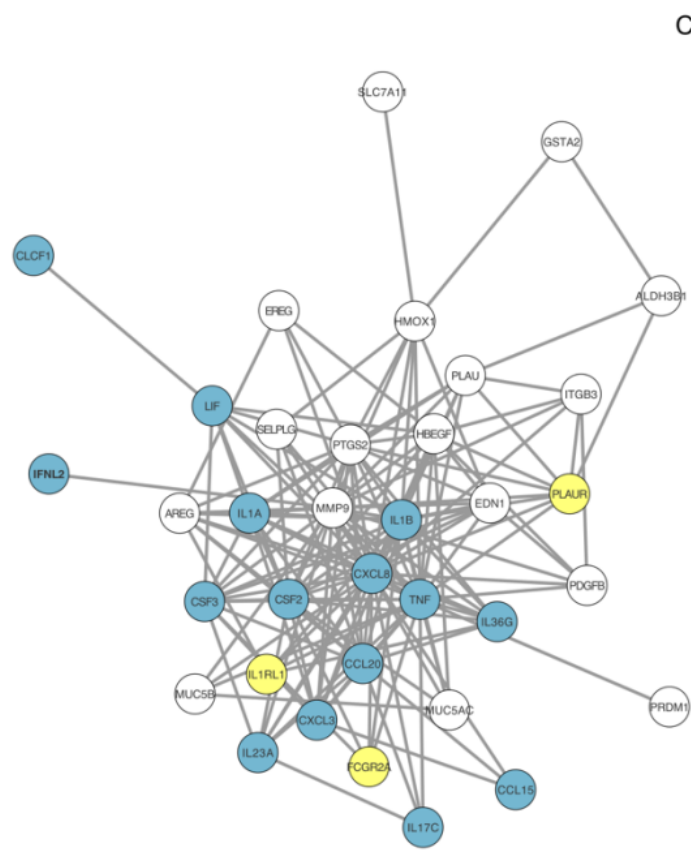

Cytokines/chemokines Receptors nasal 


\section{Figure 3}

528 (A) \& (B) Network visualization of immunity-associated proteins in superinfected HAE. We 529 selected the 76 and 191 genes significantly differentially expressed (vs. CoV) in our study 530 (respectively in nasal (A) and bronchial (B) HAE) associated with the Reactome "Interleukin53110 signaling" (R-HSA-6783783) and "cytokine signaling in immune system" pathway (R-HAS532 1280215), the Uniprot keyword "inflammatory response" (KW-0395) and the Gene Ontology 533 term "immune system process" (GO:0002376). The circles indicate genes modulated in our 534 study in the superinfection context. The predicted associations are materialized by the dark 535 lines. Cytokines/chemokines were highlighted in blue and receptors in yellow. 
Extended Data

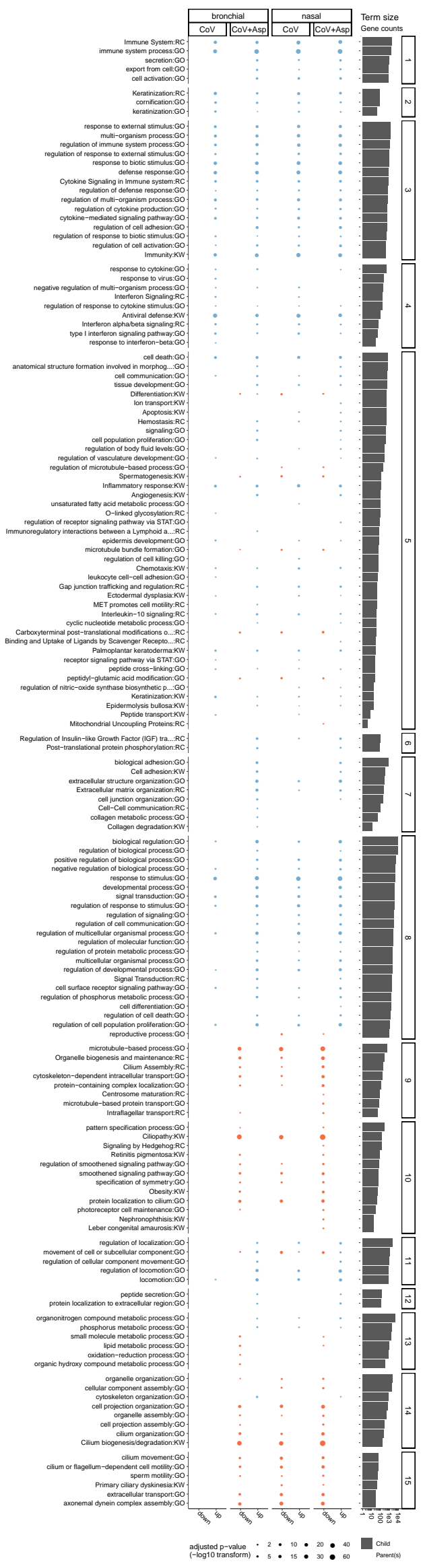




\section{Extended Data Figure 1}

539 Complete view of functional enrichment results for SARS-CoV2 or SARS-CoV-2+Aspergillus

540 infected conditions vs. Mock. Considering (CoV vs. Mock) and (CoV+Asp vs. Mock) for both

541 bronchial and nasal epithelium type, significantly up- or down- regulated gene lists (x-axis)

542 were tested for significant enrichment using the parent-child strategy (see methods). If below

543 the threshold (0.01), the adjusted $\mathrm{p}$-values corresponding to different terms ( $\mathrm{y}$-axis) are

544 represented by point sizes (see legend). Terms were clustered based on gene occurrences

545 (binary distance \& Ward algorithm) in 15 metagroups. The bar plot on the right represents the

546 sizes of enriched terms (called child) in comparison to the size of their parents (see methods

547 for definitions). 
bioRxiv preprint doi: https://doi.org/10.1101/2020.05.19.103630; this version posted May 19, 2020. The copyright holder for this preprint (which was not certified by peer review) is the author/funder. All rights reserved. No reuse allowed without permission.

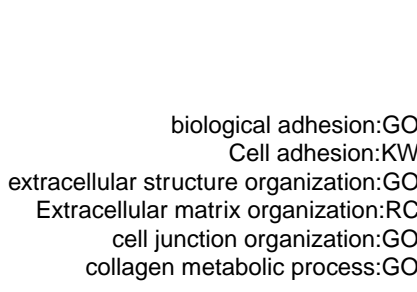

Regulation of Insulin-like Growth Factor (IGF) tra...:RC Post-translational protein phosphorylation:RC -

anatomical structure formation involved in morphog...:GO regulation of cell adhesion:GO Differentiation:KW Hemostasis:RC

regulation of cell activation:GO regulation of peptidyl-tyrosine phosphorylation:GO

Angiogenesis: KW -

biological regulation:GO positive regulation of biological process:GO negative regulation of biological process:GO response to stimulus:GO developmental process:GO-

regulation of response to stimulus:GO signal transduction:GOregulation of signaling:GO

regulation of cell communication:GO regulation of multicellular organismal process:GO

multicellular organismal process:GO regulation of developmental process:GO Signal Transduction:RC cell surface receptor signaling pathway:GOregulation of cell death:GO regulation of cell population proliferation:GO reproductive process:GO

Ion transport:KW rhythmic process:GO Inflammatory response: $\mathrm{KW}$. microtubule bundle formation:GO Chemotaxis: KW -

Gap junction trafficking and regulation:RC Interleukin-10 signaling:RC

Carboxyterminal post-translational modifications o...:RC Binding and Uptake of Ligands by Scavenger Recepto...:RC endodermal cell differentiation:GO Epidermolysis bullosa:KW -

Immune System:RC immune system process:GO regulation of immune system process:GO defense response:GO Cytokine Signaling in Immune system:RC Immunity:KW -

cell projection organization:GO microtubule-based process:GO Organelle biogenesis and maintenance:RC Cilium biogenesis/degradation:KW cytoskeleton-dependent intracellular transport:GO Ciliopathy: $\mathrm{KW}$ protein localization to cilium:GO -

regulation of localization:GO movement of cell or subcellular component:GO regulation of cellular component movement:GOregulation of locomotion:GO locomotion:GO -

cell communication:GO signaling:GO -

cilium movement:GO Primary ciliary dyskinesia:KW extracellular transport:GO axonemal dynein complex assembly:GO
Term size

Gene counts

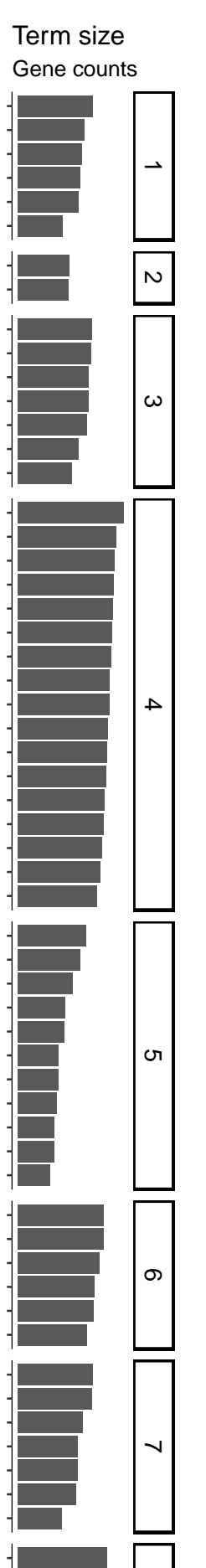

N
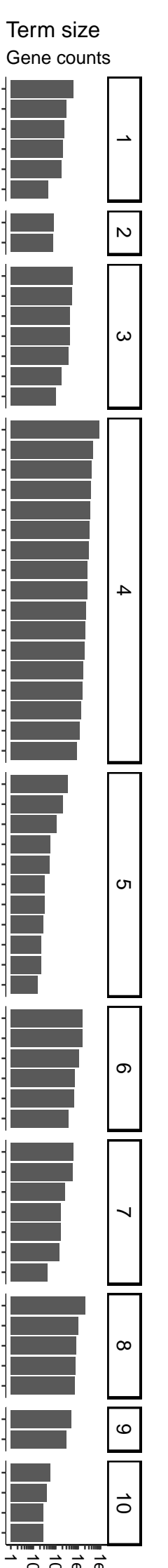

Child

Parent(s) 


\section{Extended Data Figure 2}

550 Complete view of functional enrichment results of SARS-CoV-2+Aspergillus superinfection vs.

551 SARS-CoV2 infection. Considering (CoV+Asp vs. CoV) for both bronchial and nasal epithelium

552 type, significantly up- or down- regulated gene lists (x-axis) were tested for significant

553 enrichment using the parent-child strategy (see methods). If below the threshold (0.01), the

554 adjusted $p$-values corresponding to different terms ( $y$-axis) are represented by point sizes (see

555 legend). Terms were clustered based on gene occurrences (binary distance \& Ward algorithm)

556 in 10 metagroups. The bar plot on the right represents the sizes of enriched terms (called child)

557 in comparison to the size of their parents (see methods for definitions).

558

\section{Extended Data File 1 \& 2:}

560 Extended data files will be available upon request olivier.terrier@univ-lyon1.fr 


\begin{tabular}{|c|c|c|}
\hline Database & Information type & URLS \\
\hline Reactome & Details (name,Id) & https://reactome.org/download/current/ReactomePathways.txt \\
\hline Reactome & $\begin{array}{l}\text { gene-term } \\
\text { associations }\end{array}$ & $\begin{array}{l}\text { https://www.uniprot.org/uniprot/?sort=score\&desc=\&compress } \\
=\text { no\&query=organism:\%22Homo\%20sapiens } \% 20[9606] \% 22 \& f \\
\text { l=\&format=tab\&force=yes\&columns=id,entry\%20name,length,g } \\
\text { enes(PREFERRED),reviewed, database(Reactome) }\end{array}$ \\
\hline Reactome & Topology & $\begin{array}{l}\text { https://reactome.org/download/current/ReactomePathwaysRel } \\
\text { ation.txt }\end{array}$ \\
\hline $\begin{array}{l}\text { Uniprot } \\
\text { Keywords }\end{array}$ & Topology & $\begin{array}{l}\text { http://ftp.ebi.ac.uk/pub/databases/interpro/ParentChildTreeFile. } \\
\text { txt }\end{array}$ \\
\hline $\begin{array}{l}\text { Uniprot } \\
\text { Keywords }\end{array}$ & Details (name,Id) & http://ftp.ebi.ac.uk/pub/databases/interpro/entry.list \\
\hline $\begin{array}{l}\text { Uniprot } \\
\text { Keywords }\end{array}$ & $\begin{array}{l}\text { gene-term } \\
\text { associations }\end{array}$ & $\begin{array}{l}\text { https://www.uniprot.org/uniprot/?sort=score\&desc=\&compress } \\
\text { =yes\&query=organism:\%22Homo\%20sapiens } \% 20[9606] \% 22 \& \\
\text { fil=\&format=tab\&force=yes\&columns=id,entry\%20name,length, } \\
\text { database(InterPro),genes(PREFERRED),reviewed }\end{array}$ \\
\hline $\begin{array}{l}\text { Gene } \\
\text { Ontology }\end{array}$ & $\begin{array}{l}\text { Topology and } \\
\text { Details (name,ld) }\end{array}$ & $\begin{array}{l}\text { https://www.uniprot.org/keywords/?sort=\&desc=\&compress=ye } \\
\text { s\&query=\&fil=\&format=obo\&force=yes' > } \\
\text { keywords/uniprot_keywords.obo.gz }\end{array}$ \\
\hline $\begin{array}{l}\text { Gene } \\
\text { Ontology }\end{array}$ & $\begin{array}{l}\text { gene-term } \\
\text { associations }\end{array}$ & $\begin{array}{l}\text { https://www.uniprot.org/uniprot/?query=organism\%3A\%22Hom } \\
\text { o\%20sapiens\%20\%5B9606\%5D\%22\&sort=score\&columns=id } \\
\% 2 \text { Centry\%20name\%2Ckeywords\%2Cgenes(PREFERRED)\% } \\
\text { 2Cdatabase(GenelD)\%2Creviewed\&format=tab }\end{array}$ \\
\hline
\end{tabular}

\section{Extended Data Table 1}

564 List of URLs parsed to build the databases used for enrichment analysis. Three types 565 of information were used (i) details about terms meaning term ID and complete name 566 and eventually category (e.g Biological process or Molecular function for Gene 567 Ontology); (ii) gene-term associations; (iii) Topology and especially child to parent's 568 relations to apply the parent-child method. 


\begin{tabular}{ccrr}
\hline $\begin{array}{c}\text { Epithelium } \\
\text { type }\end{array}$ & sample & $\begin{array}{c}\text { Percentage of fragments } \\
\text { aligned }\end{array}$ & \multicolumn{2}{c}{$\begin{array}{c}\text { Millions of fragments } \\
\text { aligned }\end{array}$} \\
\hline Bronchial & CoV+Asp & $22,30 \%$ & 9,6 \\
& CoV+Asp & $25,60 \%$ & 5,7 \\
& CoV+Asp & $25,20 \%$ & 7,1 \\
& CoV & $61,50 \%$ & 13,8 \\
& CoV & $66,30 \%$ & 15,9 \\
& CoV & $46,00 \%$ & 11,5 \\
& Mock & $80,30 \%$ & 19,1 \\
& Mock & $78,60 \%$ & 20,9 \\
& Mock & $79,20 \%$ & 18,5 \\
& CoV+Asp & $45,40 \%$ & 10,8 \\
& CoV+Asp & $47,00 \%$ & 15,1 \\
& CoV+Asp & $40,90 \%$ & 9,5 \\
& CoV & $53,00 \%$ & 15,2 \\
& CoV & $53,00 \%$ & 14,4 \\
& CoV & $57,10 \%$ & 15,7 \\
& Mock & $82,20 \%$ & 21,7 \\
& Mock & $80,70 \%$ & 22,1 \\
& Mock & $80,40 \%$ & 19,2 \\
\hline
\end{tabular}

572 Statistics of RNA-Seq fragment pseudo-alignment to the human transcriptome. 